\title{
Implementation and Evaluation of the Integrated Acupuncture and Moxibustion Treatment (Acupuncture, Moxibustion and Auricular Acupuncture) for Major Depressive Disorder: A Trial Protocol for a Randomized Clinical Trial
}

\section{Yuan Zhang}

2nd Clinical Medical College of Guangzhou University of Chinese Medicine: Guangdong Provincial Hospital of Traditional Chinese Medicine https://orcid.org/0000-0001-7652-4132

\section{Yamin Liu}

Guangzhou University of Traditional Chinese Medicine: Guangzhou University of Chinese Medicine

\section{Baile Ning}

2nd Clinical Hospital of Guangzhou Chinese Traditional Medicine College: Guangdong Provincial Hospital of Traditional Chinese Medicine

\section{Luda Yan}

Guangzhou University of Traditional Chinese Medicine: Guangzhou University of Chinese Medicine Lihua Wu

Guangzhou University of Traditional Chinese Medicine: Guangzhou University of Chinese Medicine Delong Zhang

South China Normal University

Changhong Li

South China Normal University

\section{Wenwei Ouyang}

Guangdong Provincial Hospital of Traditional Chinese Medicine

\section{Shengyong Su}

GuangXi University of Chinese Medicine

\section{Shuo Jiang}

Zhejiang University of Traditional Chinese Medicine First Affiliated Hospital: Zhejiang Hospital of Traditional Chinese Medicine

\section{Guangcai Zhang}

Hainan Province Hospital of Traditional Chinese Medicine Junfeng Xu

Tianjin University of Traditional Chinese Medicine

\section{Zhen Wang}


Anhui University of Traditional Chinese Medicine

\section{Zhong Zheng}

West China Hospital of Medicine: Sichuan University West China Hospital

\section{Dong Zheng}

Affiliated Brain Hospital of Guangzhou Medical University: Guangzhou Huiai Hospital

\section{Shan Chen}

Guangzhou University of Traditional Chinese Medicine: Guangzhou University of Chinese Medicine

\section{Lu Sun}

Guangdong Provincial Hospital of Traditional Chinese Medicine

\section{Wenbin Fu ( $\nabla$ fuwenbin@gzucm.edu.cn )}

2nd Clinical Medical College of Guangzhou University of Chinese Medicine: Guangdong Provincial Hospital of Traditional Chinese Medicine

\section{Study protocol}

Keywords: acupuncture, moxibustion, auricular acupuncture, integrative treatment, Major depressive disorder, sertraline

Posted Date: August 20th, 2021

DOI: https://doi.org/10.21203/rs.3.rs-801003/v1

License: (c) (1) This work is licensed under a Creative Commons Attribution 4.0 International License. Read Full License 


\section{Abstract}

Background: Because of inadequate psychotherapy resources, antidepressants are the front-line option for Major depressive disorder, but with dissatisfied curative effect. Many patients have an interest in acupuncture and moxibustion treatment as complementary therapies. However, there is no sufficient evidence on effectiveness of the integrated treatment. The aim of this study is to evaluate the efficacy and safety of the integrated acupuncture and moxibustion treatment in treating major depressive disorder and to find out the most suitable implementation protocol.

Methods: This study is a multicenter, single-blind, $2 \times 2$ factorial randomized trial. A total of 592 adult participants with moderate depression will be enrolled and randomized into four groups with a ratio of 2 : 2: $2: 1$, that is, combined group, integrated acupuncture and moxibustion treatment only group, sertraline only group, or placebo group. Participants will undergo a 12-week intervention with either $50 \mathrm{mg}$ of sertraline or placebo once a day, as well as the active/sham integrated acupuncture and moxibustion treatment for three times per week. The primary outcome is assessed using the 17-item version of Hamilton Depression Scale. The secondary outcomes are anxiety and sleep-related scales that assessed using Hamilton Anxiety Scale and Pittsburgh Sleep Quality Index. Safety will be evaluated using liver and kidney function tests examined before and after treatment, as well as daily adverse events. The primary and secondary outcomes will be measured at weeks $0,4,8,12$ and 8-week posttreatment, and an intentto-treat principle will be followed for outcomes analyses.

Discussion: Sertraline is one of antidepressants as the front-line treatment for major depressive disorder, but with a delayed onset, high rate of inadequate response and low rate of adherence. As a traditional Chinese therapy, acupuncture and moxibustion are popular and widely accepted. This trial will provide more sufficient evidence for the efficacy and safety of the integrated treatment for major depressive disorder and the most suitable combination strategy. Thus, these finds will extend our knowledge in treating for major depressive disorder.

Trial registration: Chinese Clinical Trial Registry ChiCTR2100042841; Registered on 30 January 2021.

\section{Contributions To The Literature}

1. We describe a $2 \times 2$ factorial design trial with a primary focus on comparing the effectiveness and safeness of the integrated Acupuncture and Moxibustion with antidepressants compared with the integrated Acupuncture and Moxibustion alone in clinical practices for people with moderate depression. The Quantitative and standardized operation is highlighted as a key element to ensure replicability of the integrated Acupuncture and Moxibustion treatment practice.

2. Study findings will demonstrate whether the integrated Acupuncture and Moxibustion treatment can be delivered as part of routine primary care for depression to enhance treatment outcomes and will determine a standardized protocol of integrated Acupuncture and Moxibustion treatment to implementation and popularization. 


\section{Background}

Depressive disorders are common mental disorders worldwide, with in up to $4.4 \%$ of the global population suffered from, and $4.2 \%$ in China[1]. Major depressive disorder (MDD) is the most prevalent depressive disorder and is a leading cause of global disease burden[2]. It is predicted by 2030 to be the leading cause of the total global disability-adjusted life years (DALY)[3]. And if left untreated, MDD can lead to suicide consequences with risk of 2.2-15\%[4]. Thus, MDD is recognized a major public health issue, having a substantial impact on individuals, on families and on society $[5,6]$.

MDD is characterized by lasting dysphoric mood, loss of interest or enjoyment, and fatigue or reduced energy. People with MDD often suffer from anxiety symptoms, sleep disturbance, cognitive impairment, or other somatic symptoms[7-9]. Currently, our treatment goals focus on symptoms controlled, achieving remission and restoration of patients' function to baseline level[10]. The effective treatments for MDD recommended in guidelines are a range of psychological interventions such as behavioural activation, cognitive behavioural therapy (CBT), counselling and interpersonal psychotherapy (IPT), as well as pharmacological interventions such as first-generation antidepressants (FGAs) and second-generation antidepressants (SGAs). However, a survey based on 17 countries found that the untreated rates of mental disorders are very high in low- and middle-income countries, between $76 \%$ and $85 \%[11]$. In China, the number of those who received treatment for depression was even less than $10 \%$ in four provinces[12].

On the one hand, reasons for low treated rates are inadequate medical resources, social stigma associated with mental disorders, and concerns about drug adverse reactions or over-reliance on medication[12], which lead to people have less chance or motivation to receive any mental therapy and are barriers to effective treating. On the other hand, due to lack of professional psychologists, particularly in low- and middle-income countries, antidepressants are used more frequently than psychological interventions[13]. And SGAs, such as selective serotonin reuptake inhibitors (SSRIs), are usually recommended as the front-line treatment for MDD in clinical guidelines[13-15]. Yet, there are still amounts of debates regarding the effectiveness of medication because of some limits such a delayed onset[16, 17], an inadequate response in up to $50 \%[4,18]$, and lack of adherence in $30 \%[19]$. These limitations are also the barriers to effective treating. In nowadays, more people showed a preference for complementary therapies[12, 20].

Acupuncture is known as one of the most representative Traditional Chinese Medicine (TCM) therapy and commonly applied as an important part of clinical practice in China[21]. Internationally, acupuncture was commonly used to treat depression and was recommended within primary care in UK[22, 23]. The American College of Physicians (ACPs) also recommended acupuncture as complementary and alternative medicine (CAM) in Clinical Practice Guideline for Adult Patients with Major depression in 2016[24]. Currently, various styles of acupuncture are applied in clinical practice, ranging from traditional/classical acupuncture to modern acupuncture such as abdominal acupuncture, auricular acupuncture and electro-acupuncture. Different styles of acupuncture may have differences in their 
advantages, and two or more acupuncture technique may applied jointly in clinical practice for diseases with complex symptoms[25]. Moxibustion is another treatment through burning moxa and produce heat to stimulate acupoints, which is most used as cooperation with acupuncture for better effect[26]. MDD is not a simple disease with single symptom, but mostly previous trials on acupuncture and treatment only focused on the outcomes of a single acupuncture therapy that is deficient in terms of integrated treatment intensity or treatment principle of MDD. Besides, although increasing accumulations of randomized trials on acupuncture and depression therapy, these studies suffer from methodological limitations and lack of high-quality experimental evidence[27].

Since 2000, we study the effect of acupuncture for depression, and formed an integrated acupuncture and moxibustion (iAM) treatment protocol called "Shugan Tiaoshen"[28]. Specifically, this treatment protocol refers to apply the conjunctions of acupuncture, moxibustion and auricular acupuncture to regulating liver function and spirit. Therefore, this study protocol is designed as a multicenter, largesample, randomized clinical test, aiming to investigate the effectiveness and safeness of this integrative therapy for MDD with moderate severity. In addition, this study is also expected to find out the best treatment strategy.

\section{Methods}

\section{Design and Setting.}

This study is conducted at nine clinical hospitals in China, where widely cover the South, East, West and North. The Primary sponsor is The Second Affiliated Hospital of Guangzhou University of Chinese Medicine (Guangdong Provincial Hospital of Chinese Medicine, GPHCM). This study will be conducted in compliance with local regulations and the international principles established in the Helsinki Declaration. Ethic approval was obtained from the Ethics Committee of GPHCM (number: BF2020-186). This trial has been registered at Chinese Clinical Trial Registry (ChiCTR2100042841).

A total of 592 MDD participants with moderate symptoms are recruited and randomly assigned in a 2 (active/sham iAM) $\times 2$ (sertraline/placebo) factorial design with a ratio of $2: 2: 2: 1$ : active iAM and sertraline (hereafter referred to as combined treatment group), active iAM and placebo (iAM only group), sham iAM and sertraline (sertraline only group), and sham iAM and placebo (placebo group).

\section{Eligibility Criteria}

In the study, inclusion criteria are showed as follow: patient is (1) diagnosed MDD guiding by The Diagnostic and Statistical Manual of Mental Disorders (DSM-V) criteria and "The Standard for TCM Diseases and Syndromes therapeutic results "which is published by Chinese TCM Authority (ZY/T001.194); (2) aged from 18 to 65 years old, with educational level is higher than junior middle school; and (3) obtained the written consent forms. The patients are excluded if they meet the below criteria: patient has (1) the Hamilton Depression Scale-17 (HAMD-17) scores $\leq 17$ or $₫ 24$; (2) suicidal tendency; (3) treatment with antidepressants over the last 6 weeks before enrollment; (4) history of psychiatric disease (e.g., 
schizophrenia, bipolar disorder, substance abuse or other mental disorders); (5) brain organic diseases, severe somatic diseases; epilepsy, or family history of strong positive epilepsy; (6) skin lesions or skin diseases, severe diabetes, tumor and important organ dysfunction or severe internal diseases; (7) has pregnancy, pregnancy preparation or lactation.

\section{Recruitment and Consent}

Participants are recruited in nine centers via local advertising, local newspapers, and the Internet. Interested individuals need to contact research assistants by phone or email and make an appointment for the first visit. During the first visit, psychological interviews and will be conducted, evaluated, and recorded according to the inclusion criteria and the exclusion criteria to ensure the eligibility of each individual. And eligible participants will be informed the trial detailed information and consent forms. Then research assistants will obtain a signed consent form from eligible participant who is and willing to participate in the trial (see Fig. 1).

\section{Randomization and Allocation}

The allocation was concealed using a central randomization method: a $2: 2: 2: 1$ permuted block randomization sequence is generated by the central randomization system of the Second Affiliated Hospital of Guangzhou University of Chinese Medicine, which divides the eligible participants into either combined treatment group, iAM only group, sertraline only group, or placebo group. The whole process of allocation is performed by a specific staff.

\section{Blinding}

The participants, data managers, and statisticians are blinded to the treatment allocations, which will not be revealed until the end of study. However, acupuncturists need to know the treatment allocations. Participants will be given the corresponding tube attached a label including the name outside, which was filled with either drugs or placebo pills. And the placebo pills are identical in appearance to the real drugs. Each participant has the treatment of acupuncture and moxibustion in a separate room to sure the adequate concealment. Due to the nature of auricular acupuncture, participants are required that auricular acupuncture should be take off by acupuncturists rather than by themselves. In addition, acupuncturists and researchers are instructed not to communicate with participants about the possibility of their allocations.

\section{Intervention}

Participants will receive the active/sham integrated Acupuncture and Moxibustion treatment three times a week (each interval is greater than $24 \mathrm{~h}$ ), a total of 36 times for 12 consecutive weeks (see Fig. 2).

\section{The Integrated Acupuncture and Moxibustion Treatment and Sham method}


The integrated Acupuncture and Moxibustion (iAM) treatment protocol comprise three interventions: manual acupuncture, moxibustion and auricular acupuncture, sequentially (see Fig. 2). For manual acupuncture intervention, patients are asked to lie supine, wearing eye masks for better curative effect. After skin disinfection with $75 \%$ alcohol, acupuncture is performed at selected acupoints including Baihui (GV20), Yintang (GV29), Touwei (ST8), Jiuwei (CV15), Zhongwan (CV12), Qihai (CV6), Taichong (LR3), Hegu (LI4), Sanyinjiao (SP6) with tube needles (Hwato Suzhou Medical Instruments, Suzhou, China) (see eFigure 1, Additional File 1). Needling lifted and thrusted together with twisted to achieve "Deqi" needling sensation, which was believed to indicate effective needling in TCM theory[29]. The needles are retained for 30 minutes and then removed. For sham conditions, the same needling procedure is conducted but without percutaneous puncture by using the needles with a blunt tip instead of a shaft one, and does not require to achieve "Deqi" sensation. Detailed information about active/sham acupuncture method and needle sizes are shown in eTable 1 and eFigure 2, Additional File 1.

After acupuncture, moxa-moxibustion intervention is conducted (see Fig. 2). Patients are asked to lie prostrate, wearing eye masks still. And they will be received alternately two groups of selected acupoints to, the one group including Feishu (BL13), Geshu (BL17), Danshu (BL19), and Yongquan (KI1), and the other group including Pohu (BL42), Geguan (BL46), Yanggang (BL48), Yongquan (KI1) (see eFigure 3, Additional File 1). Wanhua oil is smeared evenly on local skin to fix moxa cones and prevent from heating. A moxa cones, with $2 \mathrm{~mm}$ in diameter and $3 \mathrm{~mm}$ in height, is placed and ignited using a joss stick (see eFigure 4, Additional File 1). When it burns off two-thirds part, remove it away. Two moxa cones are applied to each point. The difference between sham moxibustion and active moxibustion is that moxa cones are not ignited.

Finally, auricular acupuncture is performed. The auricular points of Heart point (C015), Liver point (CO12), and Kidney point (CO10) are selected for intervention (see eFigure 5A, Additional File 1). Auricular needles[30] (0.2mm in diameter, $0.6 \mathrm{~mm}$ in length; Seirin Co Ltd, Shimize-City, Japan) are used to bedded in auricular points of unilateral auricle and remained for $24 \sim 72$ hours, then teared by acupuncturists in the next treatment. Auricular acupuncture is conducted at one side for each treatment, left and right alternately. For sham conditions, sham auricular needles, where needles are not placed, are used to instead of real ones. (see eFigure $5 B$ and $C$, Additional File 1)

\section{Drugs Treatment and Placebo}

The participants in combined treatment group and sertraline only group are given a tube filled with antidepressant medicine, Sertraline (Approval number: h10980141, Pfizer Co., Ltd), and asked to take orally $50 \mathrm{mg}$ of the antidepressant medicine after breakfast, once a day, continuously for 12 weeks. The participants in the iAM only group and placebo group will receive the same dosage, administration but be given the tube filled with placebo pills instead, which are made of starch. These placebo pills are not differently from antidepressant in appearance.

Due to the changes in participants illness, no prohibitions are made for medication changes during the trial. But any changes in dose or medications must be checked by psychiatrists and are recorded. 


\section{Study Visits and Measures}

Five visits are performed in this study. The study procedures are shown in Fig. 1 and Table 1. In screening visit, patients will receive psychological interview to screen the eligibility according to inclusion \& exclusion criteria. Diagnosis of TCM Syndrome Types, demographic information and medical history are conducted. Eligible patients then undergo assessments and are collected baseline data. Three visits will occur in treatment period (weeks 4, 8, 12), and one follow-up visit at week 20. The study assessments include HAMD-17 and the Patient Health Questionnaire-9 (PHQ-9), the Hamilton Anxiety Scale (HAMA), the Pittsburgh sleep quality index (PSQI), Sleep actigraphy, safety by the adverse events report. Liver and kidney function tests are also applied to assess safety, blood sample only collected at baseline (week 0 ) and after treatment (week 12). Data collection and analysis are conducted by two research assistants who are blinded to the treatment allocations.

\section{Primary Outcome}

The 17-item version of HAMD is an established clinician-rated assessment of depressive symptom severity and encompasses psychological as well as somatic symptoms[31]. It assesses the severity of 17 depression symptom items over the past week, which has five factors of Anxiety/somatization, weight, cognitive impairment, retardation, and sleep. The higher HAMD-17 score implies more severe depressive symptoms. The severity is classified as no depression (score $\leq 7$ ), mild (8-16), moderate (17-24) and severe ( $\geq 25)$. The score less than 8 is interpreted as remission, and decreasing greater than $\geq 50 \%$ is interpreted as effective treatment response. This measure has evidence of good reliability[32, 33].

\section{Secondary Outcomes}

The PHQ-9 is a self-administered version of the Primary Care Evaluation of Mental Disorders (PRIME-MD) diagnostic instrument for depression disorders[34]. Its scores at each of criteria range from " 0 " (not at all) to "3" (nearly every day)[35]. Thus, the higher the score, the more severe the depression. It has been shown to be sensitive to change and used as a brief depression severity measure tool[36, 37]. the 14-item version of HAMA is designed to standardize clinician assessment for the level of anxiety, which scores at each item are a 5-point scale from "0" (not at all) to "4" (nearly every day). HAMA-14 classifies anxiety factors into physical symptoms and mental symptoms. The 1-6 and 14 items reflect mental anxiety, while the 7-13 items reflect somatic anxiety symptoms.

PSQI is a self-rated questionnaire to assess sleep quality and disturbances for up to a one-month period. And it has been a recommended measure for treatment effectiveness studies for global sleep quality. From 19 items, 7 sub-scores are calculated including sleep quality, sleep latency, habitual sleep efficiency, sleep duration, sleep disturbances, sleep medication use, and daytime dysfunction[38]. The sum of these components yields a global score of 21 , a higher score indicating poorer sleep quality. A PSQI score greater than five implies poor sleepers and a change in three points suggests a clinical effect. Besides, the actigraphy, with a bracelet-appearance, is used as a supplemental tool to assess the sleep quality (YWK-P9; BOZHILUN, Inc., Shenzhen, Guangdong, China). It can monitor the data of real-time 
sleep quality, sleep oxygen, blood pressure, heart rate, exercise situation and other indicators, and be used to indicate the physical condition. In our study, we use it to collect quantitative sleep data and supplement sleep quantity information. Each of participants has an actigraphy and a smartphone app connecting with the actigraphy by Bluetooth matching to collect data.

\section{Safety Assessment}

Venous blood samples will be collected by venipuncture technique for liver and kidney function examinations at baseline and post-treatment. Adverse event records will be monitored and recorded at every visit to assess the safety of the study intervenes.

\section{Quality Control}

Before recruitment, a training workshop was required to offer to the whole research team including acupuncturists and research assistants. The acupuncturists who apply the treatment should have acupuncture licenses from the Ministry of Health of the People's Republic of China and have training in the application of the integrated acupuncture and moxibustion treatment. These performances are done before the trial to ensure their strict adherence to the study protocol and familiarity with the trial administration process. They are also supplied with a written protocol and standard operation procedure documents.

The data collected in this trial from information recorded in the Case Report Forms (CRFs) and an online data management platform (eMedlnform, LinkerMedTechCo., Ltd, Beijing, China), a data recording and control system for the clinical scientific research. All modifications are marked on the CRFs and system. Data quality is checked regularly by research assistants and overseen by monitors. Audits are performed regularly by the GPHCM Department of Science Research. Data monitoring is conducted regularly adhering to standard operation procedures by the Guangdong International Clinical Research Center of Chinese Medicine (Guangzhou, China). If participants withdraw from the trial either during the treatment period or the follow-up phase, the reasons should be clarified only if they are willing and the rate is analyzed via statistics.

\section{Statistical Analysis.}

Efficacy analyses will be performed based on the intent-to-treat (ITT) principle if not stated otherwise. We defined two study populations: the intent-to-treat (ITT) and per-protocol (PP) population.

Demographic and other basic characteristics will be summarized by assigned groups. Categorical variables are summarized by frequencies and percentages. Continuous variables are summarized by both mean and standard deviation for data with normal distribution or median and inter-quartile range for nonnormally distributed data.

We intend to perform two separate analyses for the primary outcome. First, for the long-term effect, analysis of variance model with a $95 \%$ confidential interval $(\mathrm{Cl})$ is used to compare the mean change of 
the HAMD score between groups without adjusting any covariates. Second, a liner mixed model will be performed. For the secondary outcomes, a Chi-squared test is used to assess the effect of the intervention on binary outcomes. Logistic regression analysis or generalized estimating equations (GEE) is performed for categorical outcomes. The reliability, validity and item response of the questionnaires is also conducted in our analyses. Safety analyses will be performed on the safety population, which will be comprised of all randomized subjects who have been administered at least one treatment. All statistical analysis will be performed by SAS 9.3 or SPSS 18.0 software. All tests of significance will be two-sided with a maximal type I error risk of $5 \%$.

\section{Sample Size Calculation}

Based on our previous research, we use a group sequential test to compare combined treatment group, iAM only group, sertraline only group, and placebo group, hypothesizing a difference in the level of among these four groups. The sample size was calculated based on mean and SD of the 17-item version of HAMD. According to the previous pilot studies[39], the data can be considered clinically significant, i.e., a mean difference of 10.47 with a standard deviation (SD) of 6.24 scores in combined treatment group, a mean difference of 12.03 (SD: 5.78) scores in iAM only group, a mean difference of 12.17 (SD: 5.78) scores in sertraline only group, and a mean difference of 19.11 (SD: 3.92) scores in placebo group. We applied a $2: 2: 2: 1$ ratio to the four groups, sequentially. Calculated that for a statistical power of $90 \%$, with a two tailed significance level of $5 \%$, the sample size was 72 for placebo group and 144 for each of the other three groups (alpha $=.05$, beta $=.10, \mathrm{~N}=503$ ). Assuming a dropout rate of approximately $15 \%$, we will require a sample size of 85 for placebo group and 169 for each of the other three groups.

\section{Discussion}

There is a growing body of literature demonstrating that acupuncture and/or related treatment may be a safe and effective treatment for depressive disorder. This study will be one of the largest trials to date assessing the efficacy and safety of acupuncture and moxibustion in depressive disorder. In this regard, the present study will be the most comprehensive nationwide trials in participants resource, i.e., participants will be recruited in 9 clinical centers covering 7 provinces and 8 cities in China.

Though acupuncture antidepressant is widely recognized, one-single acupuncture technology can not completely solve the clinical problem due to the complexity and diversity of depressive symptoms. We formulated the integrated acupuncture and moxibustion treatment to improve the sufficient of singleacupuncture therapy. According to the different stages or severity of the disease (e.g., the acute or chronic state of illness), the integrated treatment is formulated as three steps: the first step applying acupuncture, the second step applying moxibustion, and the third step applying auricular acupuncture to consolidate and extend the curative effect. In this 4-armed clinical trial, we focus on the different efficiencies among active iAM plus antidepressant, active iAM plus placebo, sham iAM plus antidepressant, and sham iAM plus placebo for moderate depressive disorder. And then, we intend to investigate whether the iAM has the positive effect on MDD or augment effective and safety combined with sertraline. Given these 
outcomes, we can assume that this treatment program could have an important role in preventing this type of depression. In addition, this study is also expected to form a standardized, applicable integrative regimen to promote the extensive implementation in improving depressive disorders.

\section{Limitations}

This study has two limitations. First, due to the nature of intervention in clinical trial, the study cannot be conducted in an ideally blind-controlled setting. Secondly, outcomes of our study cannot be interpreted as the effective in anyone of the integrated acupuncture and moxibustion treatment.

\section{Conclusion}

This clinical trial is designed to prove the hypothesis that the integrated acupuncture and moxibustion treatment can augment effective and safety of conventional antidepressant therapy. The results will also provide more evidences about the effective and safety of the integrated acupuncture and moxibustion and lead to supplement or alterations in routine strategy.

\section{Abbreviations}

MDD: Major depressive disorder; DALY: disability-adjusted life years; CBT: cognitive behavioural therapy; IPT: interpersonal psychotherapy; FGAs: First-generation antidepressants; SGAs: second-generation antidepressants; SSRIs: selective serotonin reuptake inhibitors; TCM: Traditional Chinese Medicine; WHO: World Health Organization; ACPs: the American College of Physicians; CAM: complementary and alternative medicine; iAM: Integrated Acupuncture and Moxibustion; GPHCM: Guangdong Provincial Hospital of Chinese Medicine; DSM-V: the Diagnostic and Statistical Manual of Mental Disorders; HAMD17: Hamilton Depression Scale-17; PHQ-9: Patient Health Questionnaire-9; HAMA: Hamilton Anxiety Scale; PSQI: Pittsburgh sleep quality index; PRIME-MD: Primary Care Evaluation of Mental Disorders; CRFs: Case Report Forms; ITT: intent-to-treat; PP: per-protocol; Cl: confidential interval; GEE: generalized estimating equations.

\section{Declarations}

\section{Acknowledgements}

The authors would like to thank all the researchers and staffs participating clinical institutions for their valuable contribution to ensure the implementation of this study protocol.

\section{Authors' contributions}

YZ, BN, LS and WF designed the study protocol. YL, LY, LW and DZ assisted in the development and operationalization of the study methods. YZ, YL, BN, LY, LW and DZ wrote the grant proposal. YZ and YL drafted the manuscript. $Y Z, Y L, B N$, $L Y$, and LS participated in the coordination of the study. CL and DZ 
assisted with data collection activities. WO generated the random allocation sequences for randomization and is the trial statistician. SS, SJ, GZ, JX, ZW, ZZ, DZ and SC developed and delivered study implementation strategies in collaboration with $Y Z, Y L$, and LW. All authors critically reviewed and approved the final manuscript.

\section{Funding}

This study was supported by grant 2020B1111100007 from Special Project for Research and Development in Key areas of Guangdong Province. Dr. Fu was also supported by grants YN2019ML13 from Guangdong Provincial Hospital of traditional Chinese Medicine for Scientific and Technological Research of TCM in the Special Funding, BAZJ2018239 from Shenzhen Bao'an Research Center for Acupuncture and Moxibustion, and SZSM201806077 from Sanming Project of Medicine in Shenzhen. The views presented here are solely the responsibility of the authors and do not necessarily reflect the official policy.

\section{Availability of data and materials}

Not applicable.

\section{Ethics approval and consent to participate}

This study was approved by the Ethics Committee of the Second Affiliated Hospital of Guangzhou University of Chinese Medicine (number: BF2020-186). Informed consent is being obtained from all participants.

\section{Consent for publication}

Not applicable.

\section{Competing interests}

The other authors declare that they have no competing interests

\section{Author details}

${ }^{1}$ Department of Acupuncture and Moxibustion, the Second Affiliated Hospital of Guangzhou University of Chinese Medicine, Guangzhou, Guangdong Province, China. ${ }^{2}$ Shenzhen Bao'an Traditional Chinese Medicine Hospital, Guangzhou University of Chinese Medicine, Shenzhen, Guangdong Province, China. ${ }^{3}$ School of Psychology, South China Normal University, Guangzhou, Guangdong Province, China. ${ }^{4}$ Key Unit of Methodology in Clinical Research, Guangdong Provincial Hospital of Chinese Medicine, Guangzhou, Guangdong Province, China. ${ }^{5}$ The First Affiliated Hospital of Guangxi University of Chinese Medicine, Nanning, Guangxi Province, China. ${ }^{6}$ The First Affiliated Hospital of Zhejiang University of Chinese Medicine, Hangzhou, Zhejiang Province, China. ${ }^{7}$ Hainan Provincial Hospital of Chinese Medicine, 
Haikou, Hainan Province, China. ${ }^{8}$ The First Affiliated Hospital of Tianjin University of Chinese Medicine, Tianjin, China. ${ }^{9}$ The Second Affiliated Hospital of Anhui University of Chinese Medicine, Hefei, Anhui Province, China. ${ }^{10}$ Sleep Medical Center, West China Hospital of Sichuan University, Chengdu, Sichuan Province, China. ${ }^{11}$ Brain Hospital Affiliated Guangzhou University of Medicine, Guangzhou, Guangdong Province, China. ${ }^{12}$ Department of Psychosomatic Medicine, Guangdong Provincial Hospital of Chinese Medicine, Guangzhou, Guangdong Province, China.

\section{References}

1. World Health Organization. Depression and Other Common Mental Disorders: Global Health Estimates. Geneva, Switzerland; 2017.

2. World Health Organization. The Global Burden of Disease: 2004 Update. In. Geneva, Switzerland: WHO Press; 2008.

3. Mathers CD, Loncar D. Projections of global mortality and burden of disease from 2002 to 2030 . Plos Medicine. 2006; 3:11.

4. $\mathrm{Ng} \mathrm{CH}$, Kato T, Han C, Wang G, Trivedi M, Ramesh V, et al. Definition of treatment-resistant depression - Asia Pacific perspectives. Journal of Affective Disorders. 2019; 245:626-636.

5. Kennedy SH, Lam RW, Morris B, Grp CDW. Clinical guidelines for depressive disorders - Summary of recommendations relevant to family physicians. Canadian Family Physician. 2003; 49:489-491.

6. Demyttenaere K, Bruffaerts R, Posada-Villa J, Gasquet I, Kovess V, Lepine JP, et al. Prevalence, severity, and unmet need for treatment of mental disorders in the World Health Organization World Mental Health Surveys. Jama-Journal of the American Medical Association. 2004; 291(21):25812590.

7. Ter Meulen WG, Draisma S, van Hemert AM, Schoevers RA, Kupka RW, Beekman ATF, et al. Depressive and anxiety disorders in concert-A synthesis of findings on comorbidity in the NESDA study. Journal of affective disorders. 2021; 284:85-97.

8. Luca A, Luca M, Calandra C. Sleep disorders and depression: brief review of the literature, case report, and nonpharmacologic interventions for depression. Clinical Interventions in Aging. 2013; doi:10.2147/CIA.S47230.

9. Richardson L, Adams S. Cognitive Deficits in Patients With Depression. Jnp-Journal for Nurse Practitioners. 2018; 14(6):437-443.

10. Gelenberg AJ. Using assessment tools to screen for, diagnose, and treat major depressive disorder in clinical practice. The Journal of clinical psychiatry. 2010; doi:10.1007/s801090000086.

11. Wang PS, Aguilar-Gaxiola S, Alonso J, Angermeyer MC, Borges G, Bromet EJ, et al. Use of mental health services for anxiety, mood, and substance disorders in 17 countries in the WHO world mental health surveys. Lancet. 2007; 370(9590):841-850.

12. Phillips MR, Zhang J, Shi Q, Song Z, Ding Z, Pang S, et al. Prevalence, treatment, and associated disability of mental disorders in four provinces in China during 2001-05: an epidemiological survey. 
Lancet. 2009; 373(9680):2041-2053.

13. Cipriani A, Furukawa TA, Salanti G, Chaimani A, Atkinson LZ, Ogawa Y, et al. Comparative Efficacy and Acceptability of 21 Antidepressant Drugs for the Acute Treatment of Adults With Major Depressive Disorder: A Systematic Review and Network Meta-Analysis. Lancet. 2018; 391(10128):1357-1366.

14. American Psychiatric Association. Practice Guideline for the Treatment of Patients With Major Depressive Disorder. Washington, DC, U.S.A; 2010.

15. Qaseem A, Barry MJ, Kansagara D, Amer Coll P. Nonpharmacologic Versus Pharmacologic Treatment of Adult Patients With Major Depressive Disorder: A Clinical Practice Guideline From the American College of Physicians. Annals of Internal Medicine. 2016; 164(5):350-9.

16. Mnie-Filali O, Faure C, Lambás-Señas L, El Mansari M, Belblidia H, Gondard E, et al. Pharmacological blockade of 5-HT7 receptors as a putative fast acting antidepressant strategy. Neuropsychopharmacology. 2011; 36(6):1275-1288.

17. Gao N, Tiliwaerde M, Zheng W, Xiong J, Li X, Jin Z. Neuropharmacological and antidepressant-like effects of ZY-1408: A novel serotonin/norepinephrine reuptake inhibitor and serotonin receptor 2C antagonist. Neuropharmacology. 2021; 182:108376.

18. Fava M. Diagnosis and definition of treatment-resistant depression. Biological Psychiatry. 2003; 53(8):649-659.

19. Rossom R, Shortreed S, Coleman K, Beck A, Waitzfelder B, Stewart C, et al. ANTIDEPRESSANT ADHERENCE ACROSS DIVERSE POPULATIONS AND HEALTHCARE SETTINGS. Depression and anxiety. 2016; 33(8):765-774.

20. Jorm AF, Medway J, Christensen H, Korten AE, Jacomb PA, Rodgers B. Public beliefs about the helpfulness of interventions for depression: effects on actions taken when experiencing anxiety and depression symptoms. Australian and New Zealand Journal of Psychiatry. 2000; 34(4):619-626.

21. Robinson N, Lorenc A, Ding W, Jia J, Bovey M, Wang X. Exploring practice characteristics and research priorities of practitioners of traditional acupuncture in China and the EU-A survey. Journal of ethnopharmacology. 2012; 140(3):604-613.

22. Hopton AK, Curnoe S, Kanaan M, MacPherson H. Acupuncture in practice: mapping the providers, the patients and the settings in a national cross-sectional survey. Bmj Open. 2012; 2(1): e000456.

23. MacPherson H, Richmond S, Bland M, Brealey S, Gabe R, Hopton A, et al: Acupuncture and Counselling for Depression in Primary Care: A Randomised Controlled Trial. Plos Medicine. 2013; 10(9): e1001518.

24. Qaseem A, Barry MJ, Kansagara D, Amer Coll P. Nonpharmacologic Versus Pharmacologic Treatment of Adult Patients With Major Depressive Disorder: A Clinical Practice Guideline From the American College of Physicians. Annals of Internal Medicine. 2016; 164(5):350-359.

25. Sherman KJ, Hogeboom CJ, Cherkin DC. How traditional Chinese medicine acupuncturists would diagnose and treat chronic low back pain: results of a survey of licensed acupuncturists in Washington State. Complement Ther Med. 2001; 9(3):146-153. 
26. Tian C, Fan Y, Xu J, Huang Y, Wang W, Wang S, et al. The efficacy and safety of acupuncture and moxibustion combined with western medicine for obsessive-compulsive disorder: A protocol for systematic review and meta-analysis. Medicine. 2020; 99(35): e21395.

27. Li M, Niu J, Yan P, Yao L, He W, Wang M, et al: The effectiveness and safety of acupuncture for depression: An overview of meta-analyses. Complementary Therapies in Medicine. 2020; 50:102202.

28. Fan L, Gong J, Fu W, Chen Z, Xu N, Liu J, et al. Gender-Related Differences in Outcomes on Acupuncture and Moxibustion Treatment Among Depression Patients. Journal of Alternative and Complementary Medicine. 2015; 21(11):673-680.

29. Lai X, Zhang G, Huang Y, Tang C, Yang J, Wang S, et al. A cerebral functional imaging study by positron emission tomography in healthy volunteers receiving true or sham acupuncture needling. Neuroscience Letters. 2009; 452(2):194-199.

30. Margolin A, Kleber HD, Avants SK, Konefal J, Gawin F, Stark E, et al: Acupuncture for the treatment of cocaine addiction: a randomized controlled trial. JAMA. 2002; 287(1):55-63.

31. Hamilton M. A rating scale for depression. Journal of neurology, neurosurgery, and psychiatry. 1960; 23(1):56-62.

32. Trajkovic G, Starcevic V, Latas M, Lestarevic M, Ille T, Bukumiric Z, et al. Reliability of the Hamilton Rating Scale for Depression: A meta-analysis over a period of 49 years. Psychiatry Research. 2011; 189(1):1-9.

33. Schwab JJ, Bialow MR, Holzer CE. A comparison of two rating scales for depression. Journal of clinical psychology. 1967; 23(1):94-96.

34. Kroenke K, Spitzer RL, Williams JBW. The PHQ-9 - Validity of a brief depression severity measure. Journal of General Internal Medicine. 2001;16(9):606-613.

35. Kroenke K, Spitzer RL, Williams JBW. The PHQ-9: validity of a brief depression severity measure. Journal of General Internal Medicine. 2001; 16(9):606-613.

36. Lowe B, Schenkel I, Carney-Doebbeling C, Gobel C. Responsiveness of the PHQ-9 to psychopharmacological depression treatment. Psychosomatics. 2006; 47(1):62-67.

37. McMillan D, Gilbody S, Richards D. Defining successful treatment outcome in depression using the PHQ-9: A comparison of methods. Journal of Affective Disorders. 2010; 127(1-3):122-129.

38. Buysse DJ, Reynolds CF, 3rd, Monk TH, Berman SR, Kupfer DJ. The Pittsburgh Sleep Quality Index: a new instrument for psychiatric practice and research. Psychiatry research. 1989; 28(2):193-213.

39. Wu Q. Acupuncture for the Sleep Quality in Patients with Mild to Moderate Depression: a real world study. PhD thesis. Traditional Chinese Medicine of Guangzhou University, Acupuncture and Moxibustion Department; 2016.

\section{Tables}

Table 1 Schedule of study visits and assessments in enrolment, treatment period, and follow-up. 


\begin{tabular}{|c|c|c|c|c|c|}
\hline & \multirow[t]{2}{*}{ Screening\&Baseline } & \multicolumn{3}{|c|}{ Treatment Period } & \multirow{2}{*}{$\begin{array}{l}\text { Follow- } \\
\text { up } \\
20 \text { weeks }\end{array}$} \\
\hline & & $\begin{array}{l}4 \\
\text { weeks }\end{array}$ & $\begin{array}{l}8 \\
\text { weeks }\end{array}$ & $\begin{array}{l}12 \\
\text { weeks }\end{array}$ & \\
\hline Inclusion/exclusion criteria & $x$ & & & & \\
\hline Consent & $x$ & & & & \\
\hline Allocation & $x$ & & & & \\
\hline Active/Sham iAM intervention & & $x$ & $x$ & $x$ & \\
\hline Sertraline/placebo drug use & & $x$ & $x$ & $x$ & \\
\hline Demography & $x$ & & & & \\
\hline Medical history & $x$ & & & & \\
\hline TCM Syndrome Types & $x$ & & & & \\
\hline PHQ-9 & $x$ & $x$ & $x$ & $x$ & $x$ \\
\hline HAMD-17 & $x$ & $x$ & $x$ & $x$ & $x$ \\
\hline HAMA & $x$ & $x$ & $x$ & $x$ & $x$ \\
\hline PSQI & $x$ & $x$ & $x$ & $x$ & $x$ \\
\hline $\begin{array}{l}\text { Sleep ActiGraphy measures } \\
\text { (sleeping characteristics) }\end{array}$ & $x$ & $x$ & $x$ & $x$ & $x$ \\
\hline $\begin{array}{l}\text { Blood sample } \\
\text { (liver and kidney function } \\
\text { tests) }\end{array}$ & $x$ & & & & $x$ \\
\hline Adverse events report & & $x$ & $x$ & $x$ & $x$ \\
\hline Adherence scale & & $x$ & $x$ & $x$ & $x$ \\
\hline Medication record & & $x$ & $x$ & $x$ & $x$ \\
\hline
\end{tabular}

iAM integrated Acupuncture and Moxibustion, TCM Traditional Chinese Medicine, $P H Q-9$ Patient Health Questionnaire, HAMD-17Hamilton Depression Scale-17, HAMA Hamilton Anxiety Scale, PSQ/ the Pittsburgh Sleep Quality Index

\section{Figures}




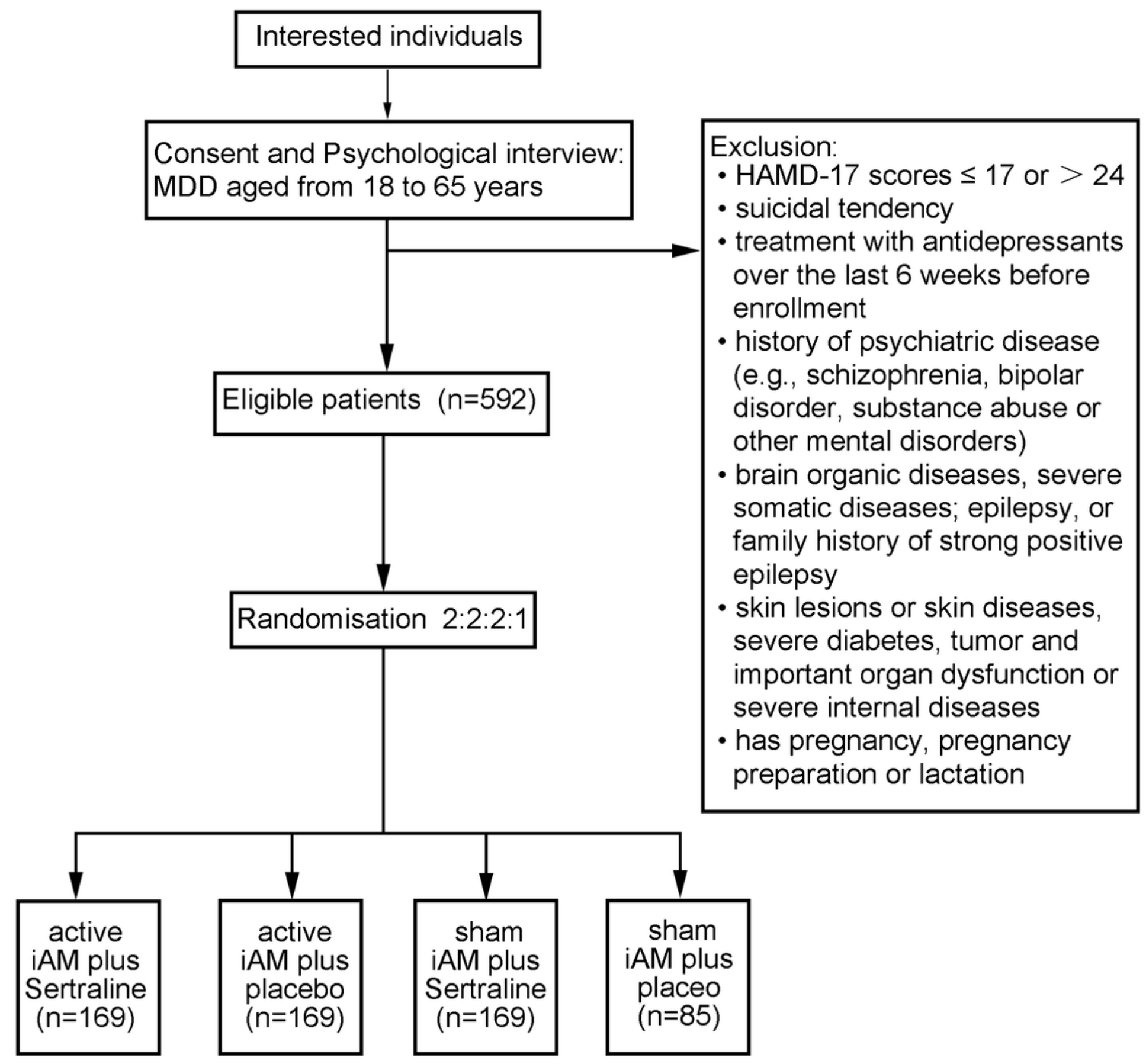

Figure 1

Study flow diagram. 


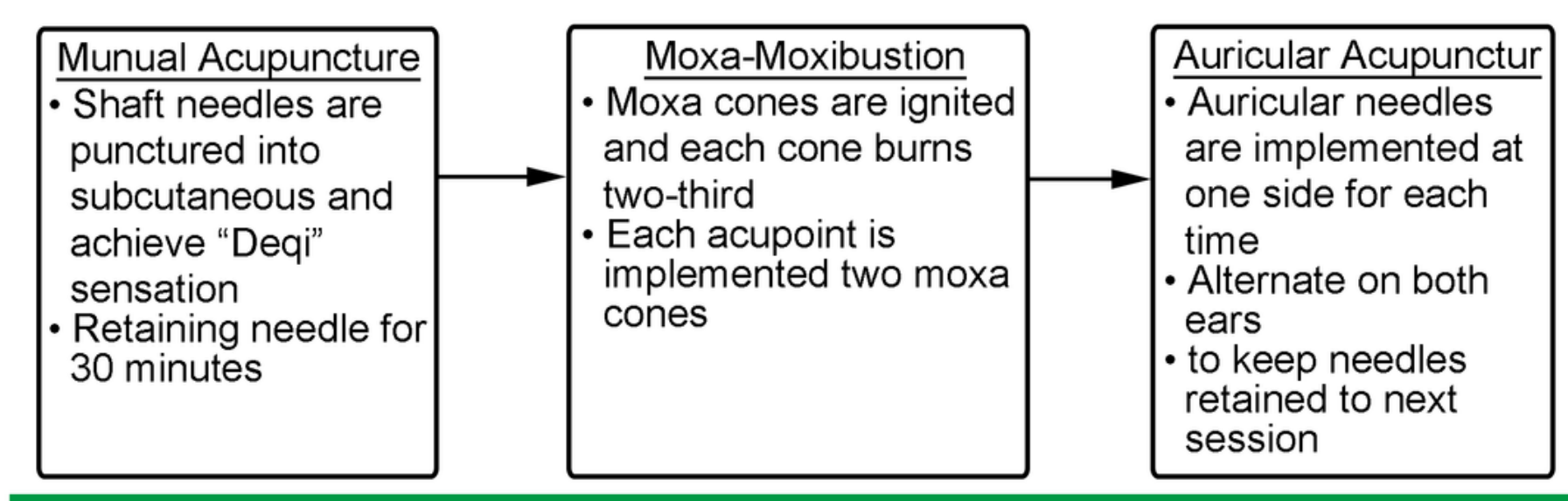

\section{A. Implementation process of active iAM intervention}

\begin{tabular}{|l|l|l|}
\hline $\begin{array}{l}\text { Munual Acupuncture } \\
\text { - Bunt needles are } \\
\text { not puncutured into } \\
\text { subcutaneous and } \\
\text { do not require to } \\
\text { achieve "Deqi" } \\
\text { sensation } \\
\text { Retaining needle for } \\
30 \text { minutes }\end{array}$ & $\begin{array}{l}\text { Moxa-Moxibustion } \\
\text { Moxa cones are not } \\
\text { ignited } \\
\text { Each acupoint is } \\
\text { implemented two moxa } \\
\text { cones }\end{array}$ \\
\cline { 2 - 3 }
\end{tabular}

\section{B. Implementation process of sham iAM intervention}

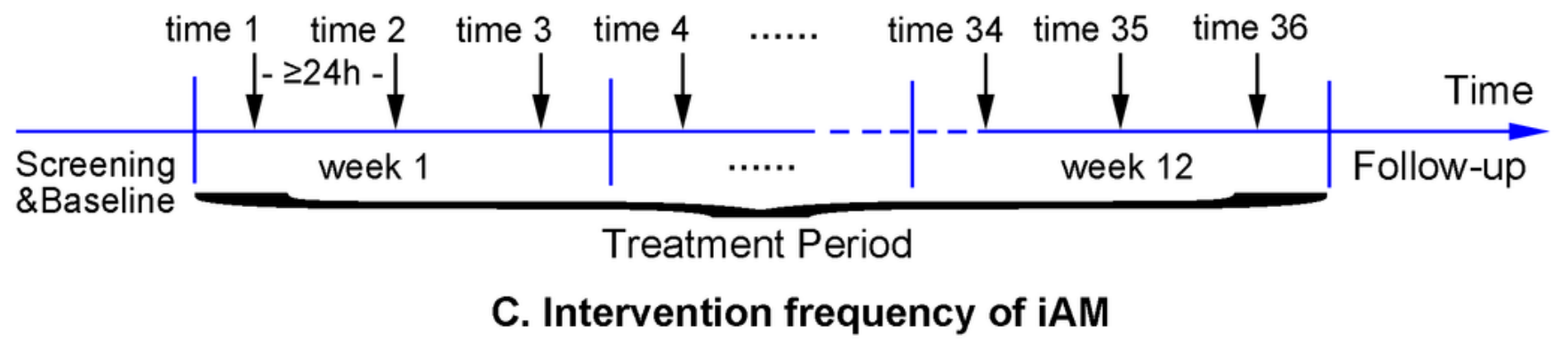

Figure 2

Implementation process of the IAM intervention. An iAM intervention contains acupuncture, moxibustion and auricular acupuncture, sequentially. Active method and sham method are presented separately in $A$ and $B$. The key differences between the two methods in process are marked by bold. Intervention frequency is presented in C: Active/Sham iAM treatment are performed three times a week (each interval is greater than $24 \mathrm{~h}$ ), a total of 36 times for 12 consecutive weeks. 


\section{Supplementary Files}

This is a list of supplementary files associated with this preprint. Click to download.

- Additionalfile1Supplements.pdf

- Additionalfile2CONSORT2010ChecklistMSWord.pdf

- Additionalfile3SPIRITFillablechecklist15Aug2013.pdf

- Additionalfile4Ethicscommitteeapproval.pdf

- Additionalfile5Fundingletter.pdf 